\title{
A Medically Challenging Case of Ganglion Impar Neurolysis in a Patient with Significant Medical Comorbidities, Coccydynia, and SeVere Disability
}

\author{
Kerolos Yousef, DO and Brian Monroe, MD
}

The term coccydynia is used to describe pain in the region of the coccyx. The majority of the cases of coccydynia occur due to chronic inflammation arising from pathologic instability and abnormal mobility of the coccyx (1). Clinical factors associated with such instability are related to obesity, trauma, childbirth, immobile coccyx leading to bursitis, and low back pain (2). Patients with high body mass index (BMIs) have a greater incidence for coccyx luxation, an abnormal mobility found in posttraumatic lesion caused by insufficient pelvic rotation while seating (3).

The ganglion impar is a solitary retroperitoneal structure anterior to the sacrococcygeal junction. It is midline and represents the caudal termination of the paravertebral sympathetic chains. It is found at a variable position in the precoccygeal space and may lie up to $2 \mathrm{~cm}$ from its classically described location anterior to the sacroccocygeal joint (4). The ganglion impar receives nociceptive inputs from visceral afferents that innervate the following structures: the perineum, the distal rectum, the anus, the distal urethra, the vulva, and the distal vagina (5). Ganglion impar block is an effective way to treat visceral and sympathetic pelvic and perineal pain of many pathologies both malignant and benign in nature (4). It is indicated to treat chronic coccydynia refractory to conservative therapy (6). Even though ganglion impar blocks are not curative, they have worthwhile palliative effects. They have high success rates for achieving substantial relief of pain, with many patients enjoying sustained relief (5).

From : Geisinger Medical Center, Danville, PA

Author for correspondence: Kerolos Yousef, DO

Address: Geisinger Medical Center, 100 North Academy Ave, Danville, PA 17822

E-mail: kyousefdo@gmail.com
In this case report, we describe a case of chronic coccydynia refractory to ganglion impar block and the use of ganglion impar neurolysis for treatment.

\section{CASE PRESENTATION}

A 63 year-old man with extensive medical history, including pulmonary embolism on chronic anti-coagulation, diabetes mellitus, hypertension, coronary artery disease, depressive mood disorder, and morbid obesity (BMI of 46.58). He had very limited mobility due to severe cervical, thoracic, and lumbar stenosis resulting in significant weakness and myelopathy. He lives at home with his wife and he requires 24 -hour care.

The patient presented to our clinic complaining of "tailbone pain." The pain was located in his lower buttocks, radiated bilaterally, and was present for over 3 months. Pain was throbbing/stabbing in nature, 9/10 in severity, exacerbated by movement and alleviated by nothing. He was unable to sit upright and any movement was excruciating, as he stated, "I can't lift my butt to put pants on." Physical therapy, chiropractic manipulation, and medications (including high dose opioids and muscle relaxants) failed to help. He was evaluated for decompressive surgical interventions and deemed to be a very high risk surgical candidate.

Initially, a diagnostic and therapeutic ganglion impar block was performed using the Foye approach (7). The patient laid in the prone position on the procedure bed. Under fluoroscopic guidance, a 22-guage, 3.5-inch spinal needle was inserted posteriorly and advanced through the sacrococcygeal junction so that the tip of the needle was just anterior to the junction. Contrast medium was injected to confirm appropriate placement anterior to the coccyx. The contrast pattern seemed neither intravascular nor too far anterior into the rectum (as shown fluoroscopically in Fig. 1). A 5 $\mathrm{mL} 0.5 \%$ bupivacaine and $80 \mathrm{mg}$ methylprednisolone 


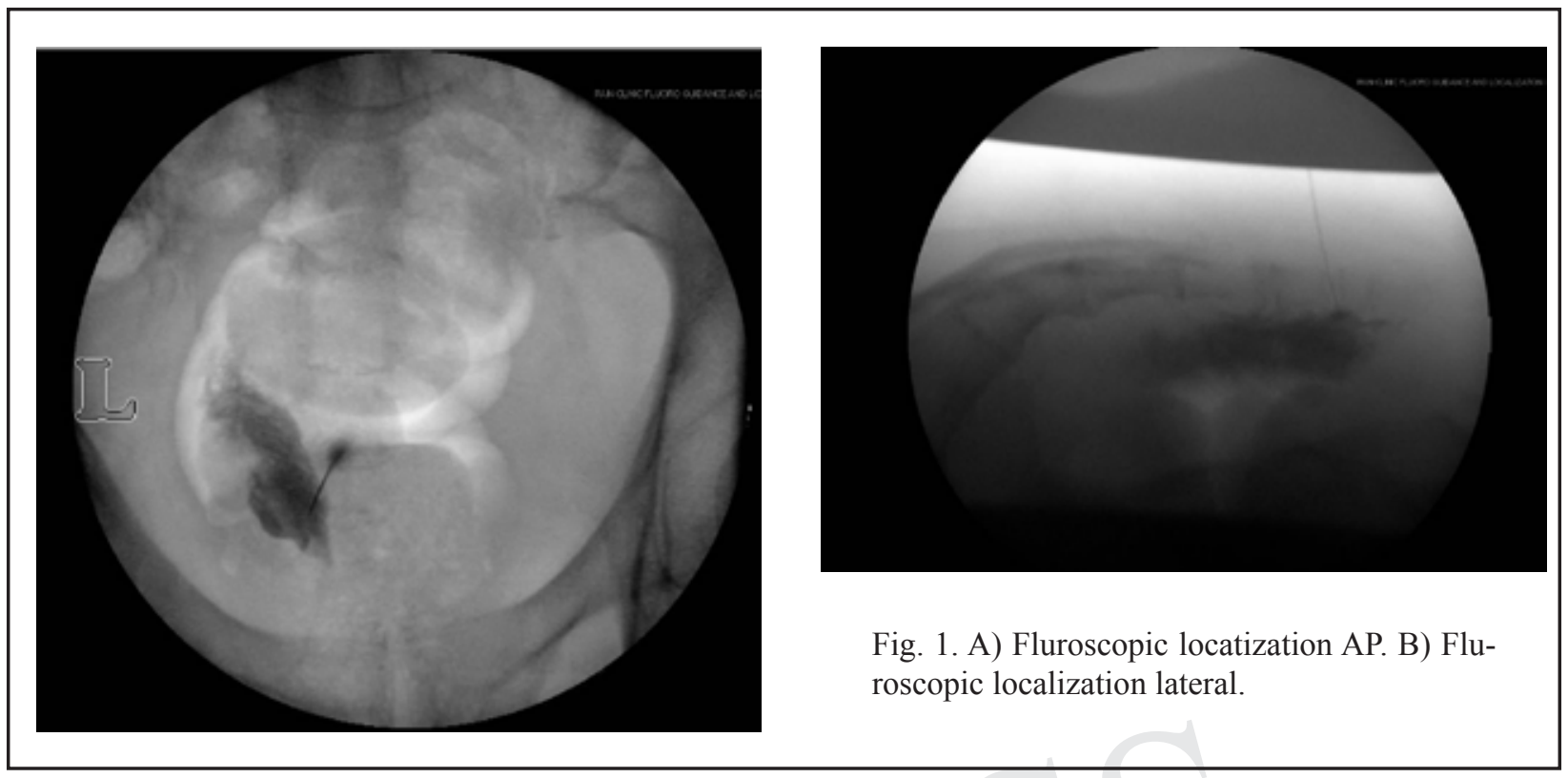

solution was injected. Patient experienced 2 days of significant improvement allowing him more mobility; however, his symptoms recurred. The same procedure was then repeated with similar results.

Due to the disabling nature of his pain, an alcohol neurolytic ganglion impar was performed. An initial dose of $4 \mathrm{~mL}$ of $1 \%$ lidocaine was injected, resulting in pain resolution within 2 minutes. Subsequently, a $4 \mathrm{~mL}$ denatured alcohol and $4 \mathrm{~mL}$ of $1 \%$ lidocaine solution was injected into the presacral space. The patient was discharged with 0 out of 10 pain level.

On follow-up, the patient reported no pain in his tailbone or low back area. He reported no loss of movement or strength compared to before the procedure. At 2 months, the patient reported continued $100 \%$ pain resolution. He was able to participate in a more intense physical therapy rehab program with an overall increase in his functional status.

\section{DISCUSSION}

Several factors have contributed to coccydynia in our patient. His elevated BMI has put him at increased risk for developing coccydynia (3). Furthermore, his chronic back pain with severe spinal stenosis worsened his ability to move. Prolonged sitting and immobility have exacerbated his coccydynia. Minor trauma from prolonged sitting on hard, narrow, or uncomfortable surfaces is an etiology for nontraumatic coccydynia (8). These factors have led to worsening luxation injury. Furthermore, his comorbid conditions further deteriorated his functional status and quality of life. After failing conservative management, ganglion impar block was the reasonable next approach. This block was initially described by Plancarte et al (9) using a spinal needle introduced through the anococcygeal ligament and directed under fluoroscopic guidance to lie with its tip retroperitoneally at the sacrococcygeal junction. With our patient, we used the Foye technique, which is done through inserting a spinal needle through the sacrococcygeal junction so that the tip of the needle reaches just anterior to the junction (7). Methylprednisolone was added to our block to help treat any potential inflammatory process that may be present as well as prolong the duration of the block (10). The block was effective, but failed to provide lasting relief.

Chemical neurolysis has been reported as an effective method to treat both malignancy related and nonmalignancy related chronic pelvic pain (11). It requires the administration of an agent that can destroy neural structures involved in the perception of pain to promote long lasting analgesia. The most common agents used for chemical neurolysis are ethyl alcohol and phenol. Ethyl alcohol has a neurodestructive effect on somatic nerves, which spares the basal lamina around the Schwann cell tube. This permits regenera- 
tion, which accounts for the return of function and pain weeks to month after the neurolytic procedure. These changes are permanent for sympathetic ganglia since the cell bodies are destroyed and regeneration is not possible; however, destruction doesn't always occur since ganglia cells are somewhat more resistant than nerve fibers. Phenol causes protein coagulation and necrosis with axonal and Wallerian degeneration. Unlike alcohol, phenol damages the neural tube and regeneration is nonspecific since there is no predetermined path for the regenerating axon to follow. There is complete necrosis of the sympathetic ganglia at 24 hours, with degeneration complete at 45 days and evidence of regeneration at 75 days, a much more rapid recovery than with alcohol (12).

In the literature, there is a report of 3 patients with rectal cancer who underwent chemical ganglion impar neurolysis (13). Over a 6 month follow-up period, these patients experienced significant pain relief, reporting a score of less than 3 on the visual analog scale (VAS), compared to a score of 9 or 10 preintervention. Another study of 6 patients with advanced pelvic malignancies who underwent ganglion impar neurolysis had significant reduction in their VAS scores and consumption of opioids while experiencing a rise in their performance status (14).

\section{REFERENCES}

1. Patel R, Appannagari A, Whang PG. "Abstract." National Center for Biotechnology Information. U.S. National Library of Medicine, 07 May 2008. Web. 21 May 2013.

2. Fogel GR, Cunningham PY 3rd, Esses SI. Coccygodynia: Evaluation and management. J Am Acad Orthop Surg 2004; 12:49-54.

3. Maigne JY, Doursounian L, Chatellier G. Causes and mechanisms of common coccydynia: Role of body mass index and coccygeal trauma. Spine (Phil Pa 1976) 2000; 25:3072-3079.

4. Scott-Warren JT, Hill V, Rajasekaran A. Ganglion impar blockade: A review. Curr Pain Headache Rep 2013; 17:306.

5. de Medicis E, de Leon-Casasola O. Ganglion impar block: Critical evaluation. Tech Reg Anesthes Pain Manag 2001; 5:120-122.

6. Gunduz $\mathrm{OH}$, Sencan S, Kenis-Coskun O. Pain relief due to transsacrococcygeal ganglion impar block in chronic coccygodynia: A pilot study. Pain Med 2015; 16:1278-1281.

7. Foye PM, Buttaci CJ, Stitik TP, Yonclas PP. Successful injection for coccyx pain. Am J Phys Med Rehabil 2006; 85:783-784.

8. Lirette LS, Chaiban G, Tolba R, Eissa H. Coccydynia: An overview of the anatomy, etiology, and treatment of coccyx pain. Ochsner ] 2014; 14:84-87.

9. Plancarte R, Amescua C, Patt RB, Allende S. Presacral blockade of the ganglion of Walther (ganglion impar). Anesthesiology 1990; 73:A751.

10. Parrington SJ, Odonnell D, Chan VW, Brown-Shreves D, Subramanyam R, Qu M, Brull R. Dexamethasone added to mepivacaine prolongs the duration of analgesia after supraclavicu-
Neurolysis is generally reserved for end of life care and patients with malignancy due to risk of surrounding nerve damage (15). In our patient, the benefit outweighed the risk since he had a very restricted functional status due to significant weakness and limited mobility. The use of chemical neurolysis allowed him to regain functionality and participate in physical therapy leading to a positive impact on his overall health condition. There have not been any reports in the literature of the use of alcohol neurolysis for chronic coccydynia due to luxation injury. It is reported in the literature the use of radiofrequency thermocoagulation of the ganglion impar for the treatment of chronic coccydynia with success in patients unresponsive to conservative treatment (16). And there is only one other case study that reported the use of alcohol neurolysis of the ganglion impar for the treatment of chronic coccydynia after spinal arachnoid cyst removal (17).

\section{CONCLUSION}

This is a very unique case of chemical neurolysis of the ganglion impar in a patient failing multiple therapies. It provides insight in its utility for select patients with chronic coccydynia refractory to conservative treatment.

lar brachial plexus blockade. Reg Anesth Pain Med 2010; 35:422426.

11. Malec-Milewska M, Horosz B, Kolęda I, Sękowska A, Kucia $\mathrm{H}$, Kosson D, Jakiel G. Neurolytic block of ganglion of Walther for the management of chronic pelvic pain. Wideochir Inne Tech Maloinwazyine 2014; 9:458-462.

12. Bonica JJ, Loeser JD. Management of Pain. (3rd ed). Lippincott Williams \& Wilkins; 2001, pp 1967-1969.

13. Eker HE, Cok OY, Kocum A, Acil M, Turkoz A. Transsacrococcygeal approach to ganglion impar for pelvic cancer pain: A report of 3 cases. Reg Anesth Pain Med 2008; 33:381-382.

14. Bhatnagar S, Khanna S, Roshni S, Goyal GN, Mishra S, Rana SP, Thulkar S. Early ultrasound-guided neurolysis for pain management in gastrointestinal and pelvic malignancies: An observational study in a tertiary care center of urban India. Pain Pract 2012; 12:23-32.

15. Gerbershagen HU. Neurolysis. Subarachnoid neurolytic blockade. Acta Anaesthesiol Belg 1981; 32:45-57.

16. Demircay E, Kabatas S, Cansever T, Yilmaz C, Tuncay C, Altinors $\mathrm{N}$. Radiofrequency thermocoagulation of ganglion impar in the management of coccydynia: Preliminary results. Turk Neurosurg 2010; 20:328-333.

17. Cha YD, Yang CW, Han JU, Song JH, Na W, Oh S, Kim BG. Transsacrococcygeal approach to ganglion impar block for treatment of chronic coccygodynia after spinal arachnoid cyst removal. Medicine (Baltimore) 2016; 95:e5010. 
